\title{
Design of genus-specific primer panel for detection and identification of viral DNA in environmental samples using next-generation sequencing
}

\author{
K. Khafizov ${ }^{1,2 *}$, A.A. Ayginin ${ }^{1,2}$, E.V. Pimkina ${ }^{1}$, A.D. Matsvai1, ${ }^{1,2}$, A.S. Speranskaya ${ }^{1,3}$, \\ M.V. Safonova ${ }^{1}$, E.A. Blinova ${ }^{1}$, I.V. Artyushin ${ }^{1,3}$, V.G. Dedkov ${ }^{1}$, G.A. Shipulin ${ }^{1}$ \\ ${ }^{1}$ Central Research Institute of Epidemiology, Moscow, Russia \\ ${ }^{2}$ Moscow Institute of Physics and Technology, Dolgoprudny, Russia \\ ${ }^{3}$ Lomonosov Moscow State University, Moscow, Russia \\ *e-mail:khafizov@cmd.su
}

Key words: virome analysis, pathogens detection, NGS

Motivation and Aim: The advances in the next generation sequencing (NGS) technologies have significantly increased our ability to detect new viral pathogens and systematically determine the spectrum of viruses that persist in various biological samples [1]. Such studies led to the discovery of new viral pathogens, as well as to establishing the associations of viromes with many diseases [2]. However, unlike the metagenomics studies using 16S rRNA for bacteria detection, it is impossible to create universal oligonucleotides to target all known and novel viruses due to the viral genomes diversity and their variability, whereas whole-genome sequencing is still expensive and relatively low-sensitive for such purposes.

Methods and Algorithms: In this study, we designed a genus-specific oligonucleotide panel for targeting enrichment of viral nucleic acids in different samples and demonstrated possibility of its application for virus detection in samples collected from migratory birds. The role of migratory birds in the circulation and spreading of a number of viral pathogens is well known [3]. Thereby the analysis of bird's seasonal migration routes and their viromes allows predicting the future directions and timing of pathogens spreading, and could help to prevent infectious outbreaks.

Results: Our panel has been tested using a number of collected bird samples and has demonstrated superior efficiency in pathogen detection and identification. The reliable bioinformatics pipeline for the rapid classification of the sequences was crucial to success of these efforts. Existing approaches for designing oligonucleotides are usually oriented to developing primers or DNA-probes for detection of particular viral species or genera [4] using PCR, but not families or higher taxonomic orders. Moreover, it is nearly impossible to design multiplex primer panel using mentioned algorithms. Thus, we developed computational pipeline for designing oligonucleotides that cover high number of known viruses belonging to different taxonomic orders and also their novel variants. In this work we also developed an NGS-based data analysis module, and demonstrated the functionality of our tool both for detecting novel viruses and for analyzing the virome diversity. Conclusion: We introduced a method for designing oligonucleotide panels for targeted viral nucleic acids enrichment, where the main idea is to use a minimal number of oligonucleotides to cover the maximal number of diverse viral taxa, and this can be performed in one PCR reaction. We applied this approach to design genus-specific primer pairs for target nucleic acids enrichment of zoonotic viruses and evaluated it using a number of samples from migratory birds. The superior increase in the viral genome coverage has been shown.

Acknowledgements: Supported by the RSF grant (17-74-20096).

References

1. Yeh Y.-T. et al. (2016) Tunable and label-free virus enrichment for ultrasensitive virus detection using carbon nanotube arrays. Sci. Advances. 2(10):e1601026.

2. Briese T. et al. (2015) Virome capture sequencing enables sensitive viral diagnosis and comprehensive virome analysis. mBio. 6(5):e01491-15.

3. Verhagen J.H. et al. (2014) Migratory birds reinforce local circulation of avian influenza viruses. PLoS One. 9(11): e112366.

4. Gall A. et al. (2008) Universal primer set for amplification and sequencing of $\mathrm{HA}_{0}$ cleavage sites of all influenza A viruses. J. Clim. Microbiol. 46(8):2561-2567. 